\title{
PARTICLE MOTION OVER THE EDGE OF AN INCLINED PLANE THAT PERFORMS AXIAL MOVEMENT IN A VERTICAL LIMITING CYLINDER
}

\author{
Serhit F. Pylypaka ${ }^{a}$, Mykola B. Klendi ${ }^{b}$, Viktor M. Nesvidomin ${ }^{a}$, \\ VIKTOR I. TROKHANIAK ${ }^{a, *}$ \\ ${ }^{a}$ National University of Life and Environmental Sciences of Ukraine, Heroiv Oborony Str., 15, Kyiv, Ukraine. \\ $b$ \\ Separated Subdivision of National University of Life and Environmental Sciences of Ukraine Berezhany \\ Agrotechnical Institute, Akademichna Str., 20, Berezhany, Ukraine. \\ * corresponding author: trohaniak.v@gmail.com
}

\begin{abstract}
Differential equations of a relative material particle motion over the edge of an inclined flat ellipse that rotates around the axis of a vertical limiting cylinder have been deduced. The position of a plane relative to the axis of the rotation is set by the angle ranging from zero to ninety degrees in its value. If the angle is equal to zero, the plane is perpendicular to the axis of rotation and if the angle is equal to ninety degrees, it passes through the axis of rotation. The equations have been solved using numerical methods. Analytical solution has been found for certain angles.

The aim of the research is to investigate the transportability of a technological material in a vertical direction by a cascade operating element that rotates in a cylindrical cover. The working part of the operating element is an inclined rigid plane, which is limited by an ellipse — the line of its contact with a cover.

The objective of the research is to analytically describe the movement of a single particle of the technological material on two surfaces, namely, an inclined plane and a vertical cover.

The research methodology is based on the methods of differential geometry and the theory of surfaces, theoretical mechanics and numerical methods of solving differential equations.

The paper presents a first developed analytical description of the relative particle motion in an ellipse - a contact line of an inclined plane and a limiting vertical cylinder, in which the inclined plane rotates. The kinematic characteristics of such motion have been determined.
\end{abstract}

KEYWORDS: inclined cylinder; oscillating motion; vertical plane; particle; differential equations; kinematic parameters.

\section{INTRODUCTION}

A particle motion over a horizontal plane in the form of a rigid disk, which rotates around a vertical axis, is the most investigated one. Such disks with blades attached to them are used in scattering centrifugal apparatuses. Operating elements with a horizontal axis of rotation in the form of a shaft with flat blades attached to it are used for scattering organic fertilizers. In addition, they can be used for mixing particles and their scattering in a centrifugal direction. The investigation of the patterns of a material particle motion over the edge of an inclined flat ellipse that rotates around the axis of a vertical limiting cylinder is interesting in terms of theory and practical use.

Major works [1, 2] consider the compound particle motion on rough surfaces of agricultural machinery operating elements. The particle motion on a horizontal disk that rotates around a vertical axis, which is either equipped with blades of the simplest designs or is without them, is analysed in these papers. Research [3] considers the particle motion on a flat disk, which rotates around the axis that is inclined to the horizon. The patterns of the particle motion on a disk without blades as well as on the one, which is equipped with rectilinear blades located in a radial direction from the axis of the rotation, are presented in this research work. Paper [4] presents the research, which is similar to ours. It considers relative particle motion at a wide range of inclination angles of a plane to the axis of rotation, beginning from a horizontal position and finishing by a vertical one. The development of a bladed operating element of a conveyer-mixer is considered in paper [5-10].

\section{MATERIAL AND METHODS}

In order to investigate the patterns of the material particle motion over the edge of an inclined flat ellipse, which rotates around the axis of a vertical limiting cylinder, let us cut a segment off an inclined plane with the help of a vertical cylinder, which is limited by an ellipse (Figure 1). Let us rotate this segment around a cylinder axis at a constant angular velocity $\omega$. If a material particle gets onto a moving segment, it will slide on it, that is to say, it will perform a relative motion. Under the action of centrifugal force, a particle will be thrown away to the inner surface 

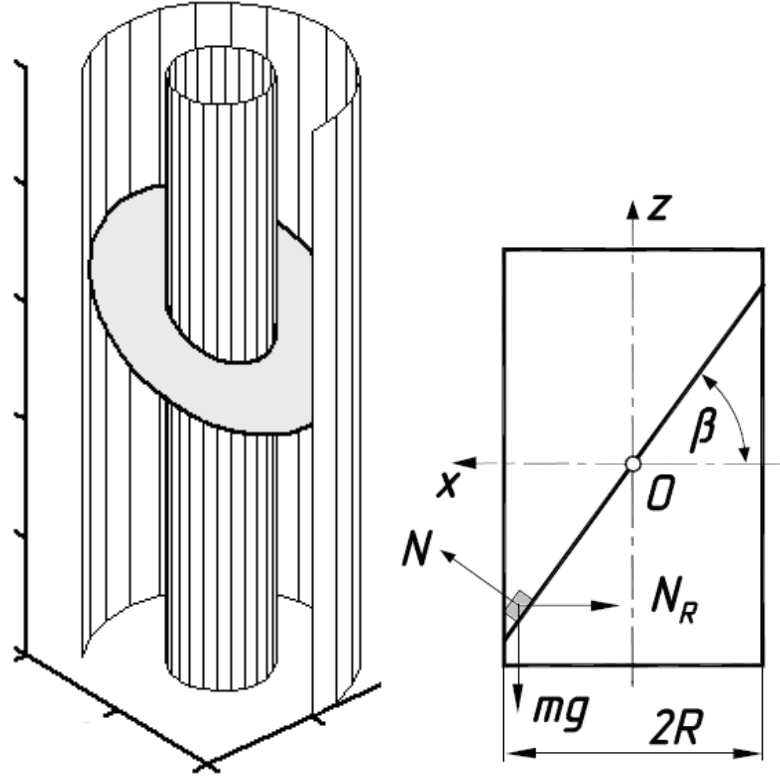

Figure 1. An inclined plane, which is limited by a vertical cylinder: (a) axonometric projection; (b) diagram of forces acting on a particle

of a limiting cylinder and, from this point on, it will move along a common intersection line of a cylinder and a plane, that is to say, along an ellipse. A particle, which is shown in Figure $1 \mathrm{~b}$ in the down position on a flat segment that is projected into a straight line, is influenced by the following forces: weight force of a particle $m g$, where $m$ - mass of a particle, $g=$ $9.81 \mathrm{~m} / \mathrm{s}^{2}$, reaction $N$ of a surface, reaction $N_{R}$ of a cylinder surface with a radius $R$. The last mentioned forces cause relative friction forces.

First of all, let us consider a situation, when a plane inclination angle is the following: $\beta=0$. Here, an ellipse will transform into a circle and the problem will become planare, which makes the problem solving much easier. Let us deduce a differential equation of an absolute particle motion in a projection onto a moving axis $s-$ motion direction (Figure 2). Let us write the equation in the following form: $m w=\sum F$, where $w$ - absolute acceleration of a particle, $\sum F-$ total force exerted upon a particle. A circular segment of a plane rotates with the angular velocity $\omega$. During the time $t$ it rotates through the angle of $\omega t$. A particle will rotate in the same direction, but its rotation will be slower, since its motion will be restricted by the friction force caused by sliding over a cylinder plane. An absolute angle of rotation will be equal to $\omega t-\alpha$, where $\alpha$ - the angle at which a particle rotates as a result of sliding on a plane (a disk). The absolute distance $s$, which a particle covers during the time $t$, is determined from the following expression:

$$
s=R(\omega t-\alpha) \text {. }
$$

Let us consider the angle $\alpha$ to be a time-varying function $t: \alpha=\alpha(t)$. With the help of the successive differentiation of the expression (1), let us find the

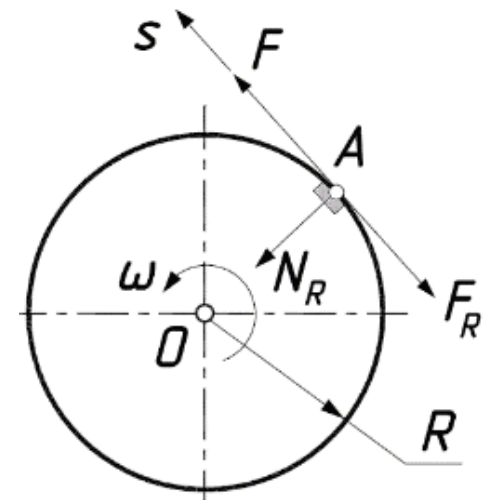

FiguRE 2. A diagram of forces acting on the particle $A$, which is located on a circular disk that rotates inside a cylindrical cover.

absolute velocity $V_{a}$ and the absolute acceleration $w$ of a particle:

$$
\begin{gathered}
V_{a}=\frac{\mathrm{d} s}{\mathrm{~d} t}=R\left(\omega-\alpha^{\prime}\right), \\
w=\frac{\mathrm{d} V_{a}}{\mathrm{~d} t}=-R \alpha^{\prime \prime} .
\end{gathered}
$$

Two friction forces, which act along the moving axis $s$ in opposite directions, are exerted upon a particle. The force $F$ of the particle friction on a disk is directed opposite to its sliding on it or in the direction of the absolute motion. Its value is the product of the coefficient of the particle friction $f$ on a disk by disk reaction $N=m g$. Thus, $F=f m g$. In a similar way, the force of the particle friction $F_{R}$ on the surface of a cylindrical cover is determined. The cover reaction on a particle $N_{R}$ balances the value of the centrifugal force $m R\left(\omega-\alpha^{\prime}\right)^{2}$ that acts on the particle. Thus, $F_{R}=f_{R} N_{R}=f_{R} m R\left(\omega-\alpha^{\prime}\right)^{2}$. After that, the equation $m w=\sum F$ takes the following form:

$$
-m R \alpha^{\prime \prime}=f m g-f_{R} m R\left(\omega t-\alpha^{\prime}\right)^{2} .
$$

After its reduction by the mass $m$ and its simplification, (4) is written as:

$$
-\alpha^{\prime \prime}=\frac{f g}{R}-f_{R}\left(\omega t-\alpha^{\prime}\right)^{2} .
$$

This equation can be analytically solved. Its partial solution can be found, which describes the particle motion after its stabilization. In this case, the angular velocity $\alpha^{\prime}$ of a particle sliding will be stable, but its angular acceleration $\alpha^{\prime \prime}$ will be equal to zero. Having solved (5) for $\alpha^{\prime}$ at $\alpha^{\prime \prime}=0$, we obtain:

$$
\alpha^{\prime}=\omega-\sqrt{\frac{f g}{f_{R} R}} .
$$

For example, at $R=0.1 \mathrm{~m}, f=f_{R}=0.3$ and $\omega=$ $20 \mathrm{~s}^{-1}$ using the formula (6), we obtain: $\alpha^{\prime}=10.1 \mathrm{~s}^{-1}$. Thus, the angular velocity of the particle rotation in the absolute motion $\omega-\alpha^{\prime}=9.9 \mathrm{~s}^{-1}$ will be twice less 
than the angular velocity of the disk rotation. The minimum value of the angular velocity of the disk rotation, at which the particle sliding is possible over its surface, is determined from the expression (6):

$$
\omega>\sqrt{\frac{f g}{f_{R} R}} .
$$

For the above mentioned parameters $\omega>9.9 \mathrm{~s}^{-1}$. If the angular velocity of the disk rotation is less than this, a particle will rotate together with it without sliding.

At $\beta \neq 0$, the absolute particle motion will be spatial. In order to find the pattern of the sliding angle change $\alpha=\alpha(t)$, let us use fixed $O x y z$ coordinates. The differential equation of motion is written in projections onto the axes of this coordinate system.

The parametrical equations of an ellipse that is created as a result of the intersection of an inclined plane and a vertical cylinder with radius $R$, are written as:

$$
\begin{gathered}
x=R \cos \alpha, \quad y=R \sin \alpha, \\
z=R \operatorname{tg} \beta \cos \alpha,
\end{gathered}
$$

where $\beta$ - the angle of the plane inclination (Figure 1p).

When a plane segment rotates with the angular velocity $\omega$, a particle in an absolute motion has to rotate in an ellipse (8). During the time $t$, an ellipse rotates through the angle $\omega t$ in the direction opposite to the particle sliding through the angle $\alpha$. Having considered the angle $\alpha$ to be sufficient, let us find the parametrical equations of an absolute particle motion. For this purpose, let us turn the ellipse 8 through the angle $-\omega t$. The coordinate $z$ does not depend on this turn, that is why, it remains unchanged. After such a turn, we obtain parametrical equations of the absolute motion of a particle:

$$
\begin{gathered}
x_{a}=R \cos \alpha \cos (-\omega t)-R \sin \alpha \sin (-\omega t) \\
=R \cos (\omega t-\alpha), \\
y_{a}=R \cos \alpha \sin (-\omega t)-R \sin \alpha \cos (-\omega t) \\
=-R \sin (\omega t-\alpha), \\
z_{a}=-R \operatorname{tg} \beta \cos \alpha .
\end{gathered}
$$

Having differentiated (9), we obtain the projections of the absolute velocity of a particle:

$$
\begin{gathered}
x_{a}^{\prime}=-R\left(\omega-\alpha^{\prime}\right) \sin (\omega t-\alpha), \\
y_{a}^{\prime}=-R\left(\omega-\alpha^{\prime}\right) \cos (\omega t-\alpha), \\
z_{a}^{\prime}=-R \alpha^{\prime} \operatorname{tg} \beta \sin \alpha .
\end{gathered}
$$

Its value is found as a geometric sum of the projections 10$)$ :

$$
\begin{aligned}
V_{a}=\sqrt{\left(x_{a}^{\prime}\right)^{2}+\left(y_{a}^{\prime}\right)^{2}+\left(z_{a}^{\prime}\right)^{2}} \\
=R \sqrt{\left(\omega-\alpha^{\prime}\right)^{2}+\left(\alpha^{\prime}\right)^{2} \operatorname{tg}^{2} \beta \sin ^{2} \alpha .}
\end{aligned}
$$

Let us find the projections of the unit vector, which sets the direction of the absolute velocity of a particle, by dividing the expressions 10 by the velocity value (11):

$$
\begin{gathered}
T_{V a x}=-\frac{\left(\omega-\alpha^{\prime}\right) \sin (\omega t-\alpha)}{A}, \\
T_{V a y}=-\frac{\left(\omega-\alpha^{\prime}\right) \cos (\omega t-\alpha)}{A}, \\
T_{V a z}=\frac{\alpha^{\prime} \operatorname{tg} \beta \sin \alpha}{A},
\end{gathered}
$$

where $A=\sqrt{\left(\omega-\alpha^{\prime}\right)^{2}+\left(\alpha^{\prime}\right)^{2} \operatorname{tg}^{2} \beta \sin ^{2} \alpha}$.

As a result of the differentiation of 10 , we obtain the projections of the absolute acceleration $w$ of a particle:

$$
\begin{gathered}
x_{a}^{\prime \prime}=R \alpha^{\prime \prime} \sin (\omega t-\alpha)-R\left(\omega-\alpha^{\prime}\right)^{2} \cos (\omega t-\alpha), \\
y_{a}^{\prime \prime}=R \alpha^{\prime \prime} \cos (\omega t-\alpha)+R\left(\omega-\alpha^{\prime}\right)^{2} \sin (\omega t-\alpha), \\
z_{a}^{\prime \prime}=R \alpha^{\prime \prime} \operatorname{tg} \beta \sin \alpha+R\left(\alpha^{\prime}\right)^{2} \operatorname{tg} \beta \cos \alpha .
\end{gathered}
$$

Let us write the equation $m w=\sum F$ in projections onto the axes of the Oxyz coordinate system. For this purpose, it is necessary to determine the forces, that act on a particle as well as their direction. Let us find the direction of the reaction force $N$ of a flat segment and the reaction force $N_{R}$ of the inner surface of a cylinder (Figure 1 $\mathrm{p}$ ). The vector force of the reaction $N$ is projected onto two axes, namely, $O x$ and $O z$. Let us write its projections on coordinate axes through the angle $\beta$ :

$$
N_{x}=N \sin \beta, \quad N_{y}=0, \quad N_{z}=N \cos \beta .
$$

The projections 14 are written without taking into consideration the rotating motion of a plane segment. In order to exert the force $N$ in the point of the particle location, the projections (14) must be turned through the angle $-\omega t$ about $O z$ axis. After this, they take the following form:

$$
\begin{gathered}
N_{\omega x}=N \sin \beta \cos \omega t, \quad N_{\omega y}=-N \sin \beta \sin \omega t, \\
N_{\omega z}=N \cos \beta .
\end{gathered}
$$

The reaction $N_{R}$ is directed towards the centre of the cylinder perpendicular to its surface, that is to say, perpendicular to the circle, which is set by the first two equations in (8). The projections of the reaction force on the coordinate axes are written as:

$$
\begin{gathered}
N_{R x}=-N_{R} \cos \alpha, \quad N_{R y}=-N_{R} \sin \alpha, \\
N_{R z}=0 .
\end{gathered}
$$

After the projections $(16)$ are turned through the angle $-\omega t$ about $O z$ axis, we obtain:

$$
\begin{gathered}
N_{R \omega x}=-N \cos (\omega t-\alpha), \quad N_{R \omega y}=0 N \sin (\omega t-\alpha), \\
N_{R \omega z}=0 .
\end{gathered}
$$

Weight force of a particle $m g$ is down-directed and does not depend on the rotation angle of a plane 
segment. Let us write its projections on the coordinate axes:

$$
(0 ; 0 ;-m g) \text {. }
$$

During an absolute particle motion, it slides both on a flat segment and on the wall of a cylindrical cover. In both cases, the value of the friction force is determined as the product of the reaction force and the correspondent friction coefficient: $F=f N$ and $F_{R}=f_{R} N_{R}$. The force $F$ is directed opposite to the direction of sliding, that is to say, opposite to the vector of the absolute velocity of particle motion. The projections of this vector are determined from differentiation of (8):

$$
\begin{gathered}
x^{\prime}=-R \alpha^{\prime} \sin \alpha, \quad y^{\prime}=R \alpha^{\prime} \cos \alpha, \\
z^{\prime}=R \alpha^{\prime} \operatorname{tg} \beta \sin \alpha
\end{gathered}
$$

Let us find the value of the relative velocity of the particle motion:

$$
V=\sqrt{\left(x^{\prime}\right)^{2}+\left(y^{\prime}\right)^{2}+\left(z^{\prime}\right)^{2}}=R \alpha^{\prime} \sqrt{1+\operatorname{tg}^{2} \beta \sin ^{2} \alpha} .
$$

The projections of a unit directing vector of relative velocity can be determined from dividing the projections 19 of this velocity by its absolute value 20]:

$$
T_{V x}=-\frac{\sin \alpha}{B}, T_{V y}=\frac{\cos \alpha}{B}, T_{V z}=\frac{\operatorname{tg} \beta \sin \alpha}{B},
$$

where $B=\sqrt{1+\operatorname{tg}^{2} \beta \sin ^{2} \alpha}$.

After the vector 21 is turned through the angle $-\omega t$, so that it is exerted to a particle, we obtain:

$$
\begin{gathered}
T_{V \omega x}=\frac{\sin (\omega t-\alpha)}{B}, \quad T_{V \omega y}=\frac{\cos (\omega t-\alpha)}{B} \\
T_{V \omega z}=\frac{\operatorname{tg} \beta \sin \alpha}{B}
\end{gathered}
$$

Here, it is possible to write the projections of the friction force $F$, taking into account that it is directed opposite to the direction of the vector 22 :

$$
\begin{gathered}
F_{\omega x}=-\frac{f N}{B} \sin (\omega t-\alpha), \quad F_{\omega y}=-\frac{f N}{B} \cos (\omega t-\alpha) \\
F_{\omega z}=-\frac{f N}{B} \operatorname{tg} \beta \sin \alpha
\end{gathered}
$$

The friction force $F_{R}$ is directed opposite to the direction of the absolute velocity, that is to say, the vector 12 . There is no need to turn the vector 12 through the angle $-\omega t$, since the cylinder is fixed and the position of a particle in an absolute motion is determined relative to a fixed coordinate system as well. Taking this into account, the projections of the force $F_{R}$ are written as follows:

$$
\begin{gathered}
F_{R x}=\frac{f_{R} N_{R}}{A}\left(\omega-\alpha^{\prime}\right) \sin (\omega t-\alpha), \\
F_{R y}=\frac{f_{R} N_{R}}{A}\left(\omega-\alpha^{\prime}\right) \cos (\omega t-\alpha), \\
F_{R z}=-\frac{f_{R} N_{R}}{A} \alpha^{\prime} \operatorname{tg} \beta \sin \alpha,
\end{gathered}
$$

Here, it is possible to write the equation $m w=\sum F$ in projections on $O x y z$ coordinate axes:

$$
\begin{array}{r}
m x_{a}^{\prime \prime}=N_{\omega x}+N_{R \omega x}+F_{x}+F_{R x}, \\
m y_{a}^{\prime \prime}=N_{\omega y}+N_{R \omega y}+F_{y}+F_{R y}, \\
m z_{a}^{\prime \prime}=N_{\omega z}+N_{R \omega z}+F_{z}+F_{R z}-m g .
\end{array}
$$

Let us substitute the expressions of the absolute acceleration 13 and the expressions of exerted forces (14), 15], 23) and (24) into (25). As a result, we obtain the system of three differential second-order equations with three unknown functions $\alpha=\alpha(t)$, $N=N(t), N_{R}=N_{R}(t)$ :

$$
\begin{gathered}
m\left(R \alpha^{\prime \prime} \sin (\omega t-\alpha)-R\left(\omega-\alpha^{\prime}\right)^{2} \cos (\omega t-\alpha)\right) \\
=N \sin \beta \cos \omega t-N_{R} \cos (\omega t-\alpha) \\
-\frac{f N}{B} \sin (\omega t-\alpha)+\frac{f_{R} N_{R}}{A}\left(\omega-\alpha^{\prime}\right) \sin (\omega t-\alpha), \\
m\left(R \alpha^{\prime \prime} \cos (\omega t-\alpha)+R\left(\omega-\alpha^{\prime}\right)^{2} \sin (\omega t-\alpha)\right) \\
=N \sin \beta \sin \omega t-N_{R} \sin (\omega t-\alpha) \\
-\frac{f N}{B} \cos (\omega t-\alpha)+\frac{f_{R} N_{R}}{A}\left(\omega-\alpha^{\prime}\right) \cos (\omega t-\alpha), \\
m\left(R \alpha^{\prime \prime} \operatorname{tg} \beta \sin \alpha+R\left(\alpha^{\prime}\right)^{2} \operatorname{tg} \beta \cos \alpha\right)=N \cos \beta \\
-\frac{f N}{B} \operatorname{tg} \beta \sin \alpha-\frac{f_{R} N_{R}}{A} \alpha^{\prime} \operatorname{tg} \beta \sin \alpha-m g .
\end{gathered}
$$

Having solved (26) for $\alpha^{\prime \prime}, N$ and $N_{R}$, we obtain:

$$
\begin{aligned}
& \alpha^{\prime \prime}=\frac{1}{m R}\left(\frac{f_{R} N_{R}}{A}\left(\omega-\alpha^{\prime}\right)-\frac{f N}{B}\right. \\
& \left.\quad+\frac{N_{R}-m R\left(\omega-\alpha^{\prime}\right)^{2}}{\operatorname{cotg}(\omega t-\alpha)}-\frac{N \sin \beta \sin \omega t}{\cos (\omega t-\alpha)}\right), \quad(27) \\
& N=D^{-1}(-4 m g A \cos \beta \\
& \left.-4 m R \sin \beta\left(\left(\alpha^{\prime}\right)^{2} A \cos \alpha+f_{R} \omega\left(\omega-\alpha^{\prime}\right)^{2} \sin \alpha\right)\right), \\
& N_{R}=D^{-1} m A\left(C R \omega\left(\omega-2 \alpha^{\prime}\right)\right. \\
& \left.\quad-R\left(3 \omega^{2}-6 \alpha^{\prime} \omega+4\left(\alpha^{\prime}\right)^{2}\right)-2 g \sin 2 \beta \cos \alpha\right),
\end{aligned}
$$

where $C=\cos 2 \alpha-2 \cos 2 \beta \cos ^{2} \alpha$ and $D=A(C-$ $3)+2 f_{R} \omega \sin ^{2} \beta \sin 2 \alpha$.

Having substituted (28) and (29) into (27), the particle mass $m$ reduces and we obtain a differential second-order equation, which can be solved using numerical methods. At $\beta=0$, the expressions $27-29$ take the following forms:

$$
\begin{gathered}
\alpha^{\prime \prime}=\frac{f_{R} N_{R}-f N}{m R}+\frac{N_{R}-m R\left(\omega-\alpha^{\prime}\right)^{2}}{m R \operatorname{cotg}(\omega t-\alpha)}, \\
N=m g, \\
N=m R\left(\omega-\alpha^{\prime}\right)^{2} .
\end{gathered}
$$

Having substituted (31) and (32) into (30), after a simplification, we obtain the differential equation (5). 
Thus, in case, when $\beta=0$, all the three equations 30 (32) completely coincide with the expressions obtained earlier, when the differential equation of an absolute particle motion was determined in the projection on a moving axis.

\section{Results}

The solution of the differential equation 27) was performed with the help of numerical methods. The investigations for a case, when $\beta=0, R=0.1 \mathrm{~m}$, $f=f_{R}=0.3, \omega=20 \mathrm{~s}^{-1}$ and when the initial integrating conditions are $\alpha^{\prime}=0, \alpha=0$, were conducted and the obtained results are presented in Figure 3

The curve in Figure 3 a shows that the angular velocity of the sliding after the motion stabilization is about $10 \mathrm{~s}^{-1}$. As a result of the analytical solution, the exact value of $\alpha^{\prime}=10.1 \mathrm{~s}^{-1}$ was obtained, which is mentioned at the beginning of the paper.

The deduced equations also work at $\omega=0$, that is to say, when there is no rotating motion of a plane segment. If the angle $\beta$ is bigger than the friction angle, a particle moves uniformly accelerated on an inclined plane. In our case, particle motion must be different, since, if an ellipse moves, the angle between a velocity vector and a horizontal plane is alternate, besides, the friction force from a cylinder wall acts on a particle. Figure 4 represents the curves of the relative velocity and the height of the particle fall movement. The initial value of the angle is $\alpha=90^{\circ}$, which corresponds to the position of a particle in the middle point through the height of a segment.

The curves show that, at first, a particle builds up speed and then it slows down its movement and stops. If a segment rotates with a low angular velocity, e.g. $\omega=5 \mathrm{~s}^{-1}$, the fall movement of a particle is different. Similar curves for such case are presented in Figure 5

The curves in Figure 5 show that there is more time needed for a particle to stop, here, at certain moments, the direction of the sliding velocity changes for the opposite one. This means that a particle performs oscillations near the low point of an ellipse and this oscillatory motion gradually declines. If the angular velocity of the segment rotation increases, a particle begins to slide in an ellipse passing through the lowest and the highest points in a sequence (Figure 60.

Having analysed the curves with the help of the similar values of the time $t$, it can be concluded that a particle has its highest sliding velocity near the lowest point and its lowest speed near the highest point. The pattern of the height change (Figure 6p) shows that a particle moves upwards slower than it moves downwards.

Let us increase the angle $\beta$ of the plane inclination to $35^{\circ}$ without changing any other parameters. In this case, a particle in a relative motion will go upwards and will stop near the highest point (Figure $7 \mathrm{a}$ ). After its stop in the relative motion, it will describe a circle in an absolute motion (Figure $7 \mathrm{~b}$ ).

The fact that a particle stops can be explained by the fact that when going upwards, there are inertial forces that make a particle move further upwards and the weight force of a particle is not enough to overcome them. Since a particle cannot move further upwards, it "sticks". It can be assumed, that such particle "sticking" can be overcome by increasing the angular velocity?. Indeed, when the angular velocity $\omega$ is increased to $24 \mathrm{~s}^{-1}$, there will be no "sticking", but, at certain moments of the particle sliding, the reaction $N$ of the surfaces will become less than zero. In Figure 8 there are two curves synchronized in time: the segment surface reaction $N$ at the particle mass $m=0.01 \mathrm{~kg}$ and the height $z$ change.

Straight vertical lines of the curves show the parts where the reaction $N$ is equal to zero or less than zero. This happens at the moment when a particle begins its fall movement. During this time, the particle detaches from a plane. A differential equation sufficiently describes the particle motion only at positive values of the reaction $N$. The physical essence of such effect can be explained by the fact that, if angular velocity is increased, inertial force increases and it overcomes the particle weight force, but here, it detaches a particle from a plane.

Let us increase the radius $R$ of a limiting cylinder to $0.25 \mathrm{~m}$ without changing any other parameters. The area of the unpredictable behaviour of a particle increases and begins even before the moment when a particle reaches its highest point at its upward movement (Figure 9).

This implies that the increase of the design parameters (the angle $\beta$ of the plane inclination, the radius $R$ of a limiting cylinder) or the technological ones (the increase of the angular velocity $\omega$ of plane rotation) causes a situation when the particle motion in an ellipse cannot be provided along the whole trajectory. There is either the particle "sticking" or its detachment from a plane.

Let us determine the role of the coefficient $f$ of the particle friction on the surface of a flat segment and the coefficient $f_{R}$ of the particle friction on the surface of a limiting cylinder. The value of these coefficients plays a part in the particle motion in an ellipse. Let us determine this motion by the dependence $z=$ $z(t)$, that is to say, by the regularity of the particle upward movement and particle fall movement. This regularity is the same for the relative motion and the absolute motion. To put that into context, let us take the curve shown in Figure $6 \mathrm{~b}$ and decrease the friction coefficient $f$ for 0.1 . Let us construct the analogical curve for the process lasting for $5 \mathrm{~s}$. This curve is presented in Figure 10 (at the top), which is synchronized in time with the surface reaction curve (at the bottom) in a similar way to the previous examples. 

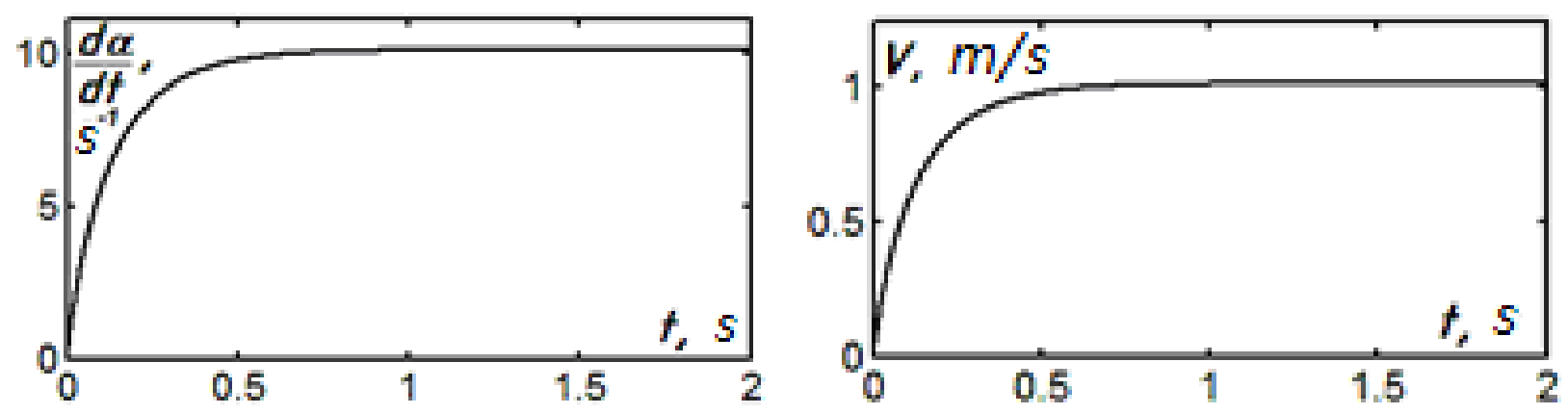

FiguRE 3. (a) Sliding angular velocity curve; (b) the curve of the relative velocity of particle sliding in a circle.
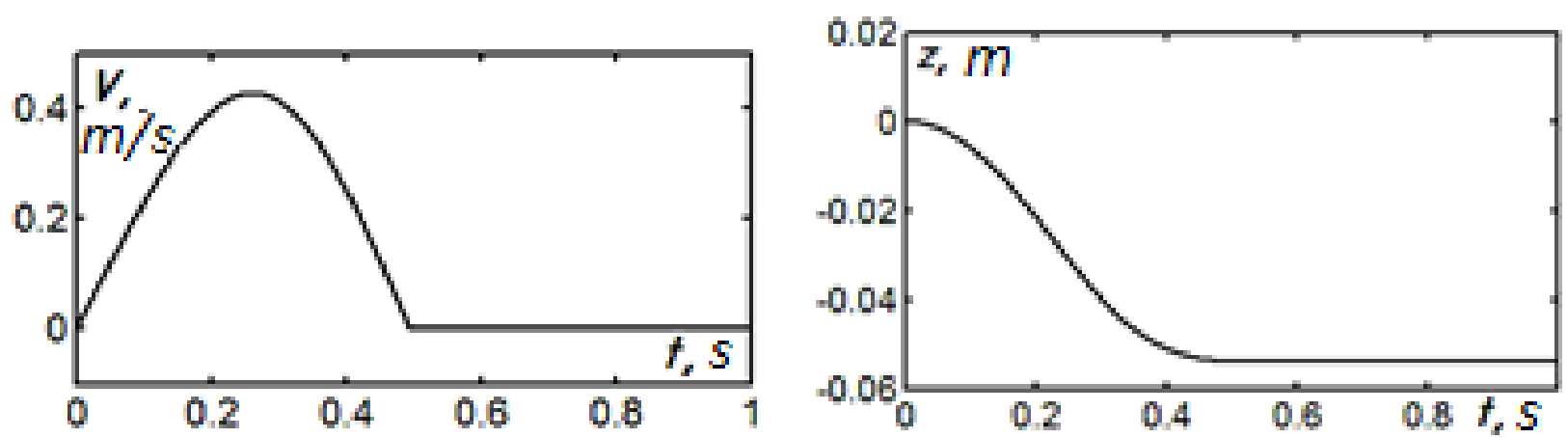

FiguRE 4. Kinematic parameter curves for particle fall movement in an ellipse of a fixed plane at $\beta=30^{\circ}, R=0.1 \mathrm{~m}$, $f=f_{R}=0.3, \omega=0$ : (a) relative velocity curve; (b) the height of particle fall movement curve.
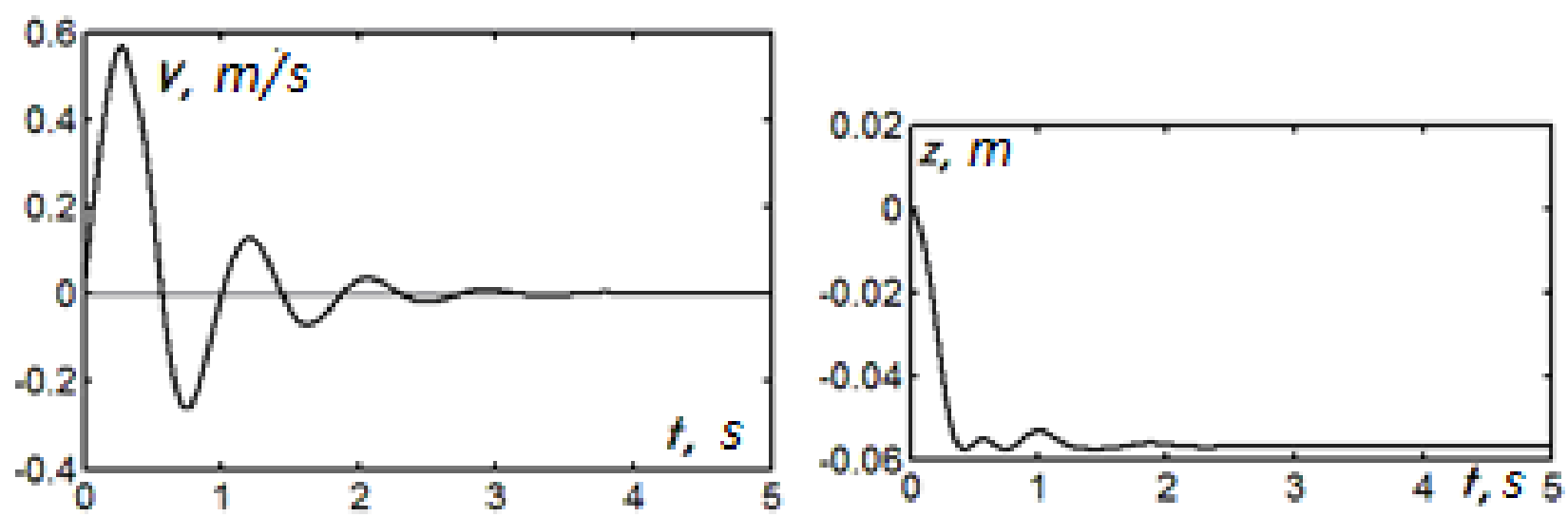

Figure 5. Kinematic parameter curves for particle fall movement in an ellipse, which rotates with low angular velocity, at $\beta=30^{\circ}, R=0.1 \mathrm{~m}, f=f_{R}=0.3, \omega=5 \mathrm{~s}^{-1}$ : (a) relative velocity curve; (b) the height of particle fall movement curve.
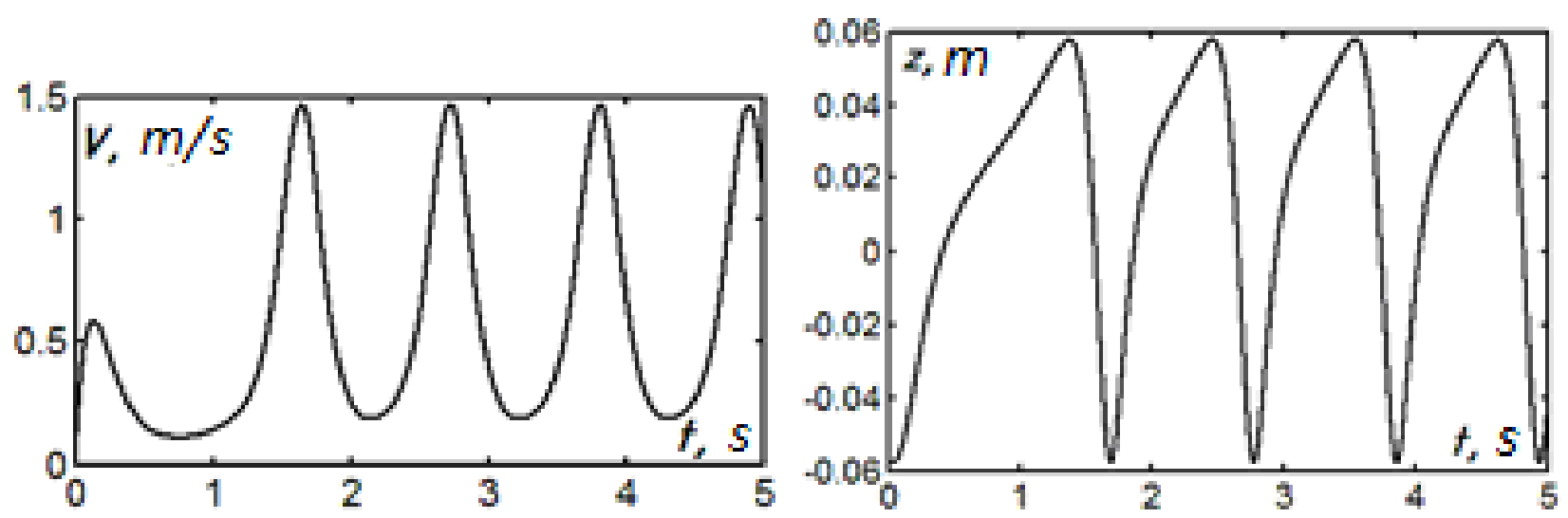

FiguRE 6. Kinematic parameter curves for particle sliding in an ellipse, which rotates with angular velocity $\omega=20 \mathrm{~s}^{-1}$, at $\beta=30^{\circ}, R=0.1 \mathrm{~m}, f=f_{R}=0.3$ : (a) relative velocity curve; (b) the height of relative motion curve. 

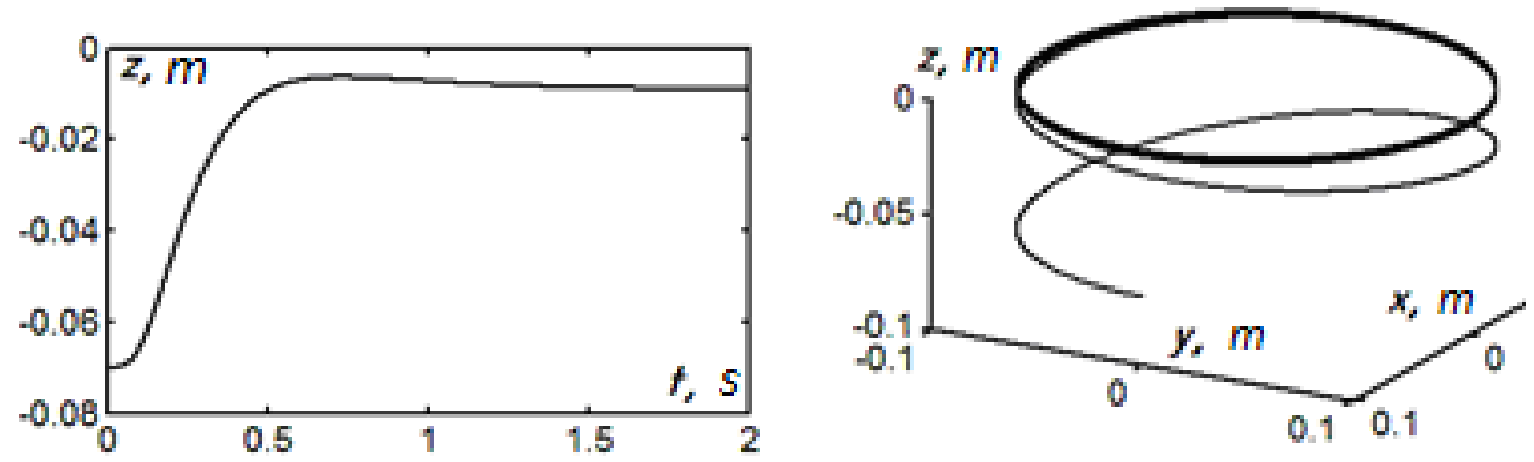

FIGURE 7. Kinematic parameter curves for particle sliding in an ellipse, which rotates with angular velocity $\omega=20 \mathrm{~s}^{-1}$, at $\beta=35^{\circ}, R=0.1 \mathrm{~m}, f=f_{R}=0.3$ : (a) the height of relative motion curve; (b) the trajectory of absolute particle motion.

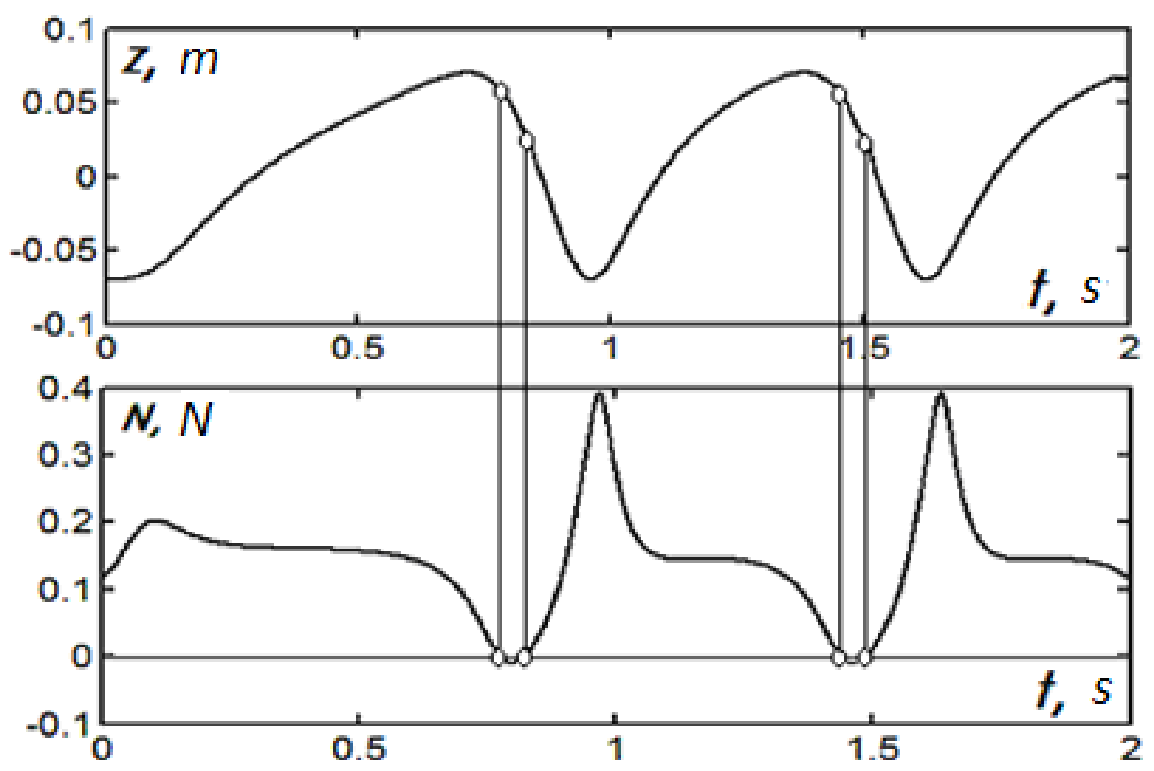

FigURE 8. The height $z$ curve (at the top) and the surface reaction $N$ curve (at the bottom) at $\omega=24 \mathrm{~s}^{-1}, \beta=35^{\circ}$, $R=0.1 \mathrm{~m}, f=f_{R}=0.3$.

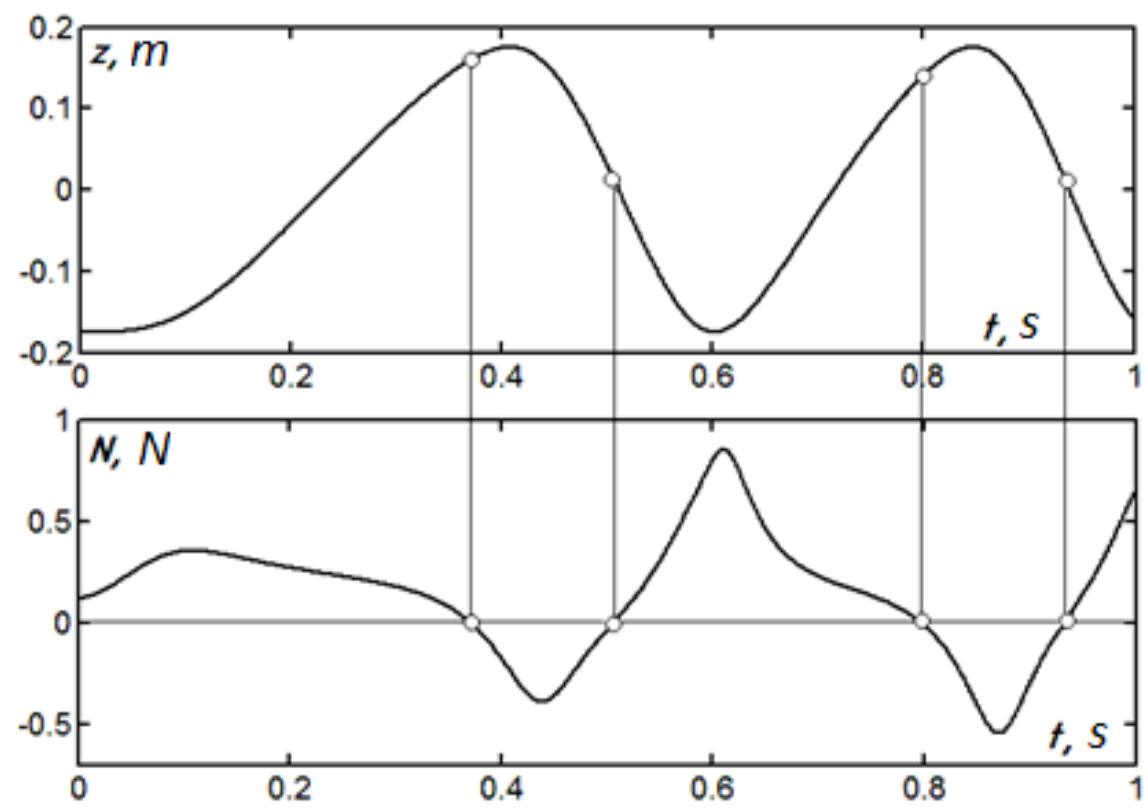

Figure 9. The height $z$ curve (at the top) and the surface reaction $N$ curve (at the bottom) at $\omega=24 \mathrm{~s}^{-1}, \beta=35^{\circ}$, $R=0.25 \mathrm{~m}, f=f_{R}=0.3$. 

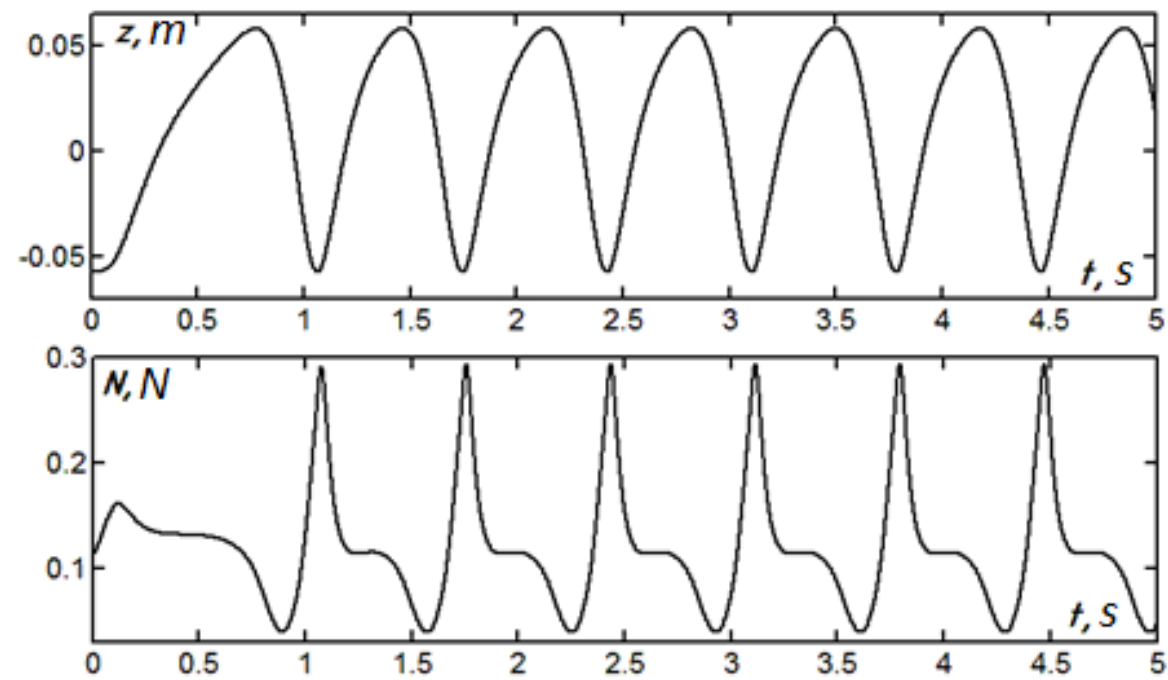

Figure 10. The height $z$ curve (at the top) and the surface reaction $N$ curve (at the bottom)at $\omega=20 \mathrm{~s}^{-1}, \beta=30^{\circ}$, $R=0.1 \mathrm{~m}, f=0.2, f_{R}=0.3$.
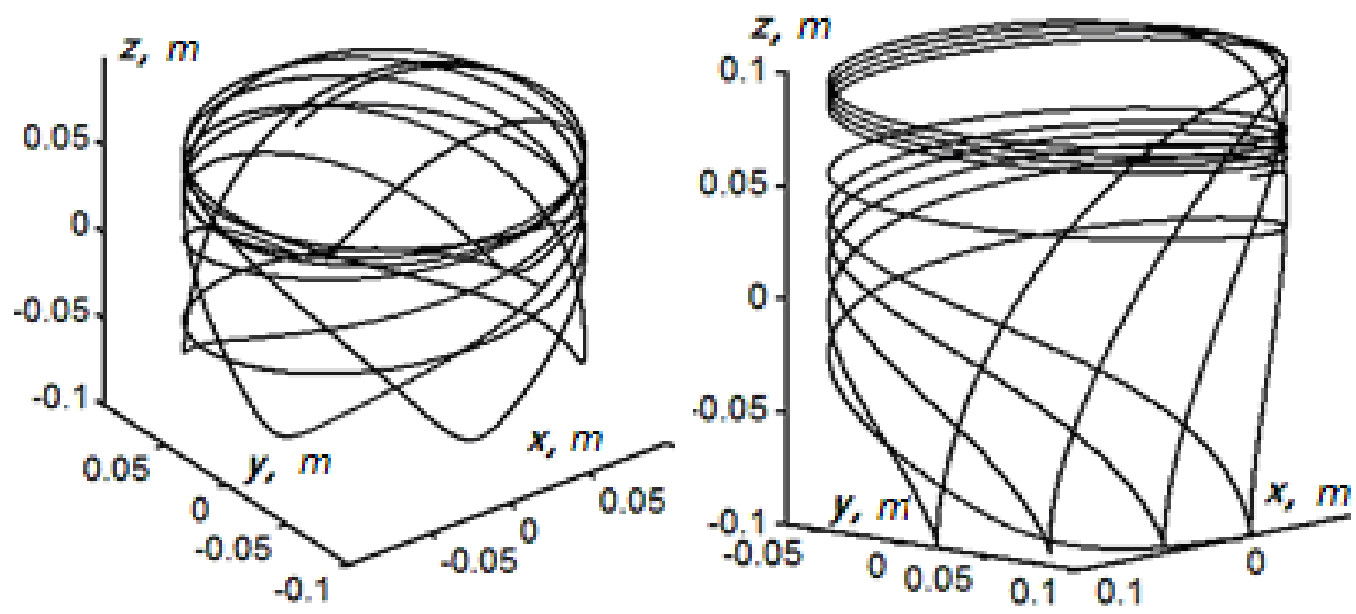

FiguRE 11. Trajectories of absolute motion over the inner surface of a limiting cylinder of radius $R=0.1$ : (a) $\omega=20 \mathrm{~s}^{-1}, \beta=30^{\circ}, f=0.2, f_{R}=0.3 ;$ (b) $\omega=18 \mathrm{~s}^{-1}, \beta=45^{\circ}, f=0.1, f_{R}=0.5$.

Having compared the curves in Figure $6 \mathrm{~b}$ and the ones in Figure 10, at the top, it can be deduced that the number of upward movements and the number of particle fall movements increased in the latter case, that is to say, the decrease of the friction coefficient $f$ resulted in the increase of the particle absolute velocity. This result is inconsistent with a plane problem at $\beta=0$. For a plane problem, the friction force $F$ between a particle and a round disk is a driving force, which pulls it in a motion in a circle in the direction of the disk rotation. The increase of the friction $f$ results in the increase of the force $F$ and, thus, the absolute velocity of the particle motion in a circle increases as well (Figure 2). By contrast, the increase of the friction coefficient $f_{R}$ results in the decrease of the absolute velocity of the particle motion in a circle, since the friction force $F_{R}$ is a decelerating force, which is directed opposite to its direction. In the case of the particle relative motion in an ellipse (at $\beta \neq 0$ ), it will be vice versa as well: the increase of the friction coefficient $f_{R}$ will increase the absolute velocity of the particle motion. At first glance, it is paradoxical, but it has its explanation. For a plane problem, the only driving force is the force $F=f N$. The reaction force $N$ is constant and it is balanced by the particle weight force $m g$. If $\beta \neq 0$, the reaction force component $N$, which is directed upwards, has the following expression $N \cos \beta$ according to the last equation in 26 . The angle cosine $\beta$ is a constant value, thus, this force is in a direct proportion to the reaction $N$. At the bottom of Figure 10, it can be seen that the force $N$ ranges widely, here, it has the highest value when a particle is located in the low point of an ellipse. It makes a particle move upwards and the decrease of the friction force, that is to say, the decrease of the coefficient $f$ will facilitate its motion. This explains the fact that the velocity of the particle sliding in an ellipse is the greatest near its low point, which has been determined as a result of the analysis of the curves presented in Figure 6. Figure 11a presents the absolute trajectory 


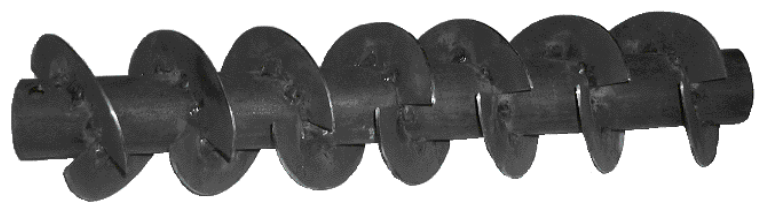

FiguRE 12. A cascade operating element.

of a particle motion. It is characterized by the abrupt change in the direction of the motion in the bottom part and a more long-term stay at the top part of an ellipse. In addition, it proves that the velocity of the particle sliding along an ellipse is greater in the bottom part than it is in the top part.

The investigations show that the increase of the friction coefficient $f_{R}$ for 0.1 results in almost the same effect as the decrease of the coefficient $f$ for the same value. If there is an increase in the coefficient $f_{R}$, the absolute angular velocity of rotation $\omega-\alpha^{\prime}$ decreases, that is to say, the value of the reaction $N_{R}$ depends on this squared value. It can be assumed that the increase of the coefficient $f_{R}$ results in the decrease of the absolute angular velocity $\omega-\alpha^{\prime}$, which square significantly influences the decrease of the decelerating force $F_{R}=f_{R} N_{R}=f_{R} m R\left(\omega-\alpha^{\prime}\right)^{2}$, whereas the coefficient $f_{R}$ increases. Thus, if there is a decrease in the friction coefficient $f$ and there is an increase in the friction coefficient $f_{R}$, the number of upward and downward movements of a particle in an ellipse is expected to be increased or there can be an increase of the angle of the plane inclination to the limits, when such motion is possible. Figure 11 p presents the trajectory of an absolute particle motion for $\beta=45^{\circ}$ at $f=0.1$ and $f_{R}=0.5$. Here, the angular velocity was decreased to $\omega=18 \mathrm{~s}^{-1}$ in order to avoid a particle detachment from the plane segment. Figure 11 p shows that the difference between the particle movement in the bottom and the top parts of an ellipse is increased even more. Particle motion is similar to its bottomupward throwing with a long-term stay at the top part of an ellipse before it moves downwards.

Taking into consideration such particle motion, several recommendations can be formulated for designing a device for lifting a technological material in a vertical pipe. In order to provide a sufficient material elevation, it is necessary to decrease the friction coefficient $f$ and to increase the friction coefficient $f_{R}$. In order to provide a continuous material feed to the specified height, a cascade operating element can be designed, as it is shown in Figure 12

Plane segments, which are limited by semi-ellipses, alternate each other, here, their inclination angles alternate in sign. The top part of the segment is cut, since there is a deceleration of the material movement.

In order to conduct the experiments, a cascade operating element for transporting loose materials by a vertical conveyer was made. The efficiency of a vertical conveyer with a screw operating element and the efficiency of a conveyer with a cascade operating element

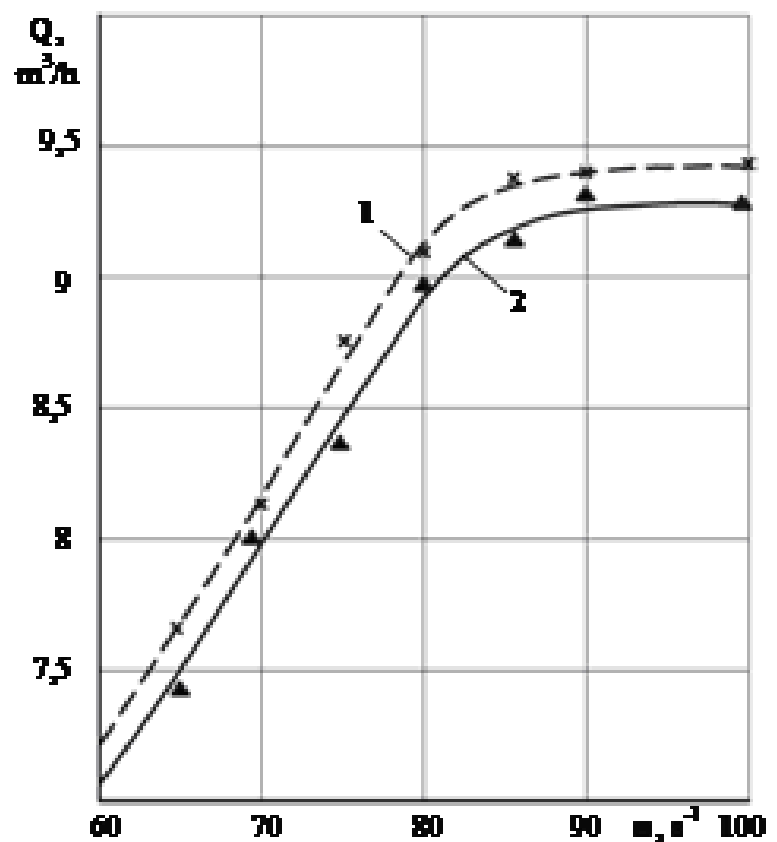

Figure 13. The efficiency of a vertical conveyer depending on the angular velocity of its operating element: 1 - a conveyer with a screw operating element; 2 - a conveyer with a cascade operating element.

was determined depending on the frequency of the rotation of an operating unit when conveying wheat $\left(f=0.3 ; f_{R}=0.3\right)$. The results of the experimental investigation are presented in Figure 13

It has been determined that the efficiency of a conveyer is at maximum and, further, it remains practically unchanged, if the angular velocity of the operating unit increases as a result of the spillage of grain through holes and gaps. The efficiency of a conveyer with a cascade operating element is practically the same as the efficiency of a conveyer with a screw operating element (error is not more than $5 \%$ ) and, that is why a cascade operating element is more appropriate to be applied in conveyers, since one of the promising directions in determining the manufacturability of screw conveyer operating elements is the application of flat blades, which are inclined to the axis of the rotation and are attached to the cylindrical shaft of a frame, instead of helical spirals. The intention is to make such blades using a sheet metal forming method and further welding them to a cylindrical shaft, since it is cheaper.

\section{Conclusions}

A particle motion on an inclined plane that rotates around the axis of a vertical limiting cylinder differs from a particle motion on a screw surface, which rotates in a vertical cylinder. The difference is in variable kinematic and dynamic motion patterns. In order to provide particle sliding on a plane and in an ellipse at the same time, it is necessary to provide the required angular velocity of the flat segment rotation. 
Its motion pattern is influenced by the coefficients of the particle friction on the surfaces of a plane and a cylindrical cover, the radius of a cylinder, the angular velocity of segment rotation and the inclination angle of a plane. At a certain combination of these parameters, a particle may "stick" in an ellipse or detach from a plane in the top position. It is possible to increase the number of particle passings along an ellipse per unit time due to the decrease of the coefficient of its friction on an inclined plane and due to the increase of the coefficient of its friction on the inner surface of a limiting cylinder. A cascade operating element, which can compete with a screw operating element in reclaim conveyers due to the ease of its manufacturing, has been designed.

\section{REFERENCES}

[1] P. M. Vasylenko, Theory of Particle Motion over Rough Surfaces of Agricultural Machines. Kyiv, Ukraine: Ukrainian Academy of Agricultural Sciences, pp. 283,1960 .

[2] P. M. Zaika, "Concerning one family of regular particle motion over an oscillating plane of vibratory grain-cleaning machine," Theory of Mechanisms and Machines, vol. 1. M. Gorkii Kharkiv State University, pp. 28-33, 1966.

[3] M. B. Klendii, O. M. Klendii. Inverrelation between incidence ange and roll ange of concave disks of soil tillage implements, INMATEH: Agricultural Engineering, vol.49, no.2, pp.13-20, 2016;

[4] Hevko R.B., Dzyura V.O., Romanovsky R.M. Mathematical model of the pneumatic-screw conveyor screw mechanism operation, INMATEH: Agricultural engineering, vol.44, no.3, pp.103-110, 2014.

[5] Pylypaka S., Klendii M., Klendii O. Particle motion on the surface of a concave soil-tilling disk, Acta Polytechnica, Journal of Advanced Engineering, Vol.58, no.3, pp.201-208, 2018.

[6] P. Ratanavararaksa and M. Dejnakarintra, "Series solutions of the anharmonic motion equation $y^{\prime \prime}+y^{2}=c$," Engineering Journal, vol. 20, no. 5, pp. 203-213, 2016.

[7] R. B. Hevko, R. I. Rozum, and O. M. Klendii, "Development of design and investigation of operation processes of loading pipes of screw conveyors," INMATEH - Agricultural Engineering, vol. 50, no. 3, pp. 89-94, 2016.

[8] Hevko B.M., Hevko R.B., Klendii O.M., Buriak M.V., Dzyadykevych Y.V., Rozum R.I. Improvement of machine safety devices. Acta Polytechnica, Journal of Advanced Engineering, Vol.58, no.1, pp.17-25, 2018.

[9] I. I. Blehman, G. Ju. Dzhenalidze. "Oscillatory displacement", Nauka, 410pp., 1964.

[10] N. I. Sysoev. Theoretical basis and calculation for a sorting device 'Zmeika', Agricultural Machinery, vol. 8, pp. 5-8, 1949. 